\title{
Coulisses
}

Revue de théâtre

43 | Automne 2011

Reviviscences de Faust

\section{Entretien avec Bachar Khalifé}

Propos recueillis par Julia Peslier

\section{Bachar Khalifé et Julia Peslier}

\section{(2) OpenEdition}

\section{Journals}

Édition électronique

URL : https://journals.openedition.org/coulisses/247

DOI : $10.4000 /$ coulisses. 247

ISSN : 2546-9460

Éditeur

Presses universitaires de Franche-Comté

\section{Édition imprimée}

Date de publication : 31 décembre 2011

Pagination : 63-71

ISBN : 978-2-84867-404-9

ISSN : $1150-594 \mathrm{X}$

\section{Référence électronique}

Bachar Khalifé et Julia Peslier, «Entretien avec Bachar Khalifé », Coulisses [En ligne], 43 | Automne

2011, mis en ligne le 30 novembre 2016, consulté le 29 décembre 2022. URL : http://

journals.openedition.org/coulisses/247 ; DOI : https://doi.org/10.4000/coulisses.247 


\title{
Entretien avec Bachar Khalifé
}

\author{
Propos recueillis par Julia Peslier
}

Bachar Khalifé et Julia Peslier

\begin{abstract}
Sur l'invitation de la Cinémathèque Française et dans le cadre de la rétrospective passionnante consacrée en 2006 au cinéma expressionniste allemand, les deux talentueux musiciens et compositeurs Bachar Khalifé (pianiste et percussionniste, nouvel album Oil Sick chez InFiné) et Rami Khalifé (pianiste, membre du trio Aufgang) ont créé une performance musicale pour le Faust de Murnau. Comment articuler leur travail de composition, d'une grande inventivité, entre formation classique, improvisation étonnante et originalité des propositions, à ce chef d'ouvre du cinéma muet, monument d'encyclopédie faustienne et fantasmagorie de l'ombre et de la lumière, où un vieillard se remet aux puissances du diable pour trouver du nouveau, renouer avec le monde qu'il a délaissé dans la bibliothèque et la médecine et réapprendre ce qui le fait homme aux côtés des autres, Esprit, peuples, puissants et belles à aimer ? Comment transformer

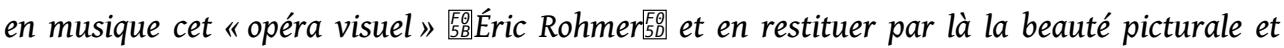
mouvante? Comment rendre sensible et intelligible sa composition extrême, afin de donner à voir et à entendre un Faust de notre temps, adressé à des spectateurs du XXI siècle? Telles seront quelques-unes des questions ici abordées.
\end{abstract}

\begin{abstract}
JULIA PESLIER - D'où vient le projet du ciné-concert autour du Faust de Murnau et quel était votre regard sur ce genre de spectacle? Comment l'avez-vous abordé au fil des performances, depuis la première invitation de la Cinémathèque Française en 2006 à composer la musique autour du Faust de Murnau, à votre création récente sur Gribiche de Feyder?
\end{abstract}

Bachar KHALIFE - Je n'avais pas une grande expérience de ce genre de spectacles. Je n'en avais jamais fait et j'avais juste assisté à un ciné-concert - je crois que c'était Le Cabinet du docteur Caligari. C'était un ami pianiste qui accompagnait un film muet. C'était quelque chose de très exotique mais en même temps, je n'imaginais pas que le ciné-concert soit le monde qu'on a découvert par la suite, c'est-à-dire un vrai monde parallèle entre le monde du cinéma et le monde de la musique. C'était un monde à part. Quand on a abordé le travail sur Faust, nous étions complètement vierges de toute expérience similaire. Je pense qu'évidemment, avec du recul, c'était une très bonne chose cette " virginité ». Du coup ce travail a été spontané, organique, je dirai. On est entré dans la psychologie du personnage, des scènes, du film, au fur et à mesure qu'on passait du temps dessus et qu'on le revoyait. C'était un travail assez 
naturel et paradoxalement, cela nous a pris beaucoup moins de temps sur Faust que sur Feyder. On avait pris la mesure de ce monde-là, on a commencé à se poser beaucoup de questions. Gribiche c'était beaucoup plus compliqué, mis à part le fait que ce sont deux films très différents. Gribiche est un film que j'ai qualifié de social, comme je l'ai dit à mon frère. C'est le cinéma français, social, sur le sentiment de l'individualisme, la solitude de ce garçon, les difficultés de la vie et d'une certaine classe sociale, alors que Faust c'est un film plus universel, qui fait appel à tous les grands mythes, que ce soit littéraire, artistique. Tout le monde l'a repris, surtout en musique. Et aussi le fait que ce soit reconnu comme un chef d'œuvre au cinéma, avec ses images, ses effets spéciaux qui n'ont pas vieilli. C'était un travail complètement différent. On a toujours eu le sentiment, en jouant Faust, d'être potentiellement en conflit avec les spectateurs qui allaient venir voir ce spectacle, peut-être justement parce qu'on n'avait pas l'habitude de ce genre de spectacle. Peut-être qu'on pensait choquer ou décevoir, en tout cas, c'est ce sentiment de conflit potentiel qui nous a donné, je pense, une force extrême pour nous confronter à ce film. Le film, quand il arrive, on met la machine jusqu'à ce qu'il se termine. Il se termine par le mot Liebe

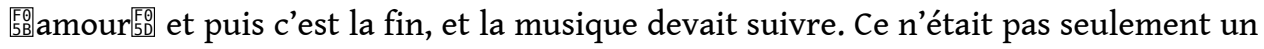
concert de musique. Quand on est musicien, sur scène, on a beaucoup de vulnérabilité. On peut être déstabilisé par un son, un bruit, par beaucoup de choses en fait, mais là, le film avance et c'est une conception très différente de faire de la musique sur scène. Plus on le joue, et plus on se rend compte que ce genre de spectacle demande beaucoup d'expérience et surtout une connaissance et un état physique assez costaud pour l'assumer.

Julia PESLIER - Quels sont vos instruments sur scène ? Comment travaillez-vous, votre frère et vous, pendant la préparation puis la mise en œuvre du ciné-concert? Quelle place accordez-vous à l'improvisation? Quelles sont les principales difficultés et les spécificités propres à la mise en musique du Faust de Murnau?

Bachar KHALIFE - Les instruments... Mon frère est pianiste, il y a un grand piano sur scène ou en fosse (cela dépend des cinémas). Moi, j'ai utilisé plusieurs instruments de percussions, notamment le cajón, des cymbales, des cloches, des sacs plastiques, mais aussi la voix. Sur la voix j'avais aussi ce qu'on appelle une loop station et des effets de distorsion, un flanger, pour la travailler et la modifier. Pour un film muet, cela peut paraître étonnant... C'est un des aspects du défi : la voix est ici utilisée comme un instrument, mais les puristes diront que c'est un blasphème d'utiliser la voix sur un film muet. On travaille toujours un peu de la même manière avec mon frère, on se connaît assez bien maintenant, on se répartit les taches naturellement selon les qualités que l'on reconnaît à l'autre. Mon frère me laisse dans un premier temps voir le film et commencer un travail sur la forme, couper les scènes - les grandes scènes et les plus petites. Faire un peu les fiches sur chaque personnage, les décrire : comment ils agissent, ce qu'ils ressentent, voilà, comme une analyse psychanalytique comprendre et apprendre. Faire vraiment la connaissance de ces personnages, dégager leurs grandes caractéristiques, découper les scènes et de là ressortir les couleurs - on parle beaucoup de couleur en musique -, et ressortir la couleur de la scène. Pour le travail sur la musique, je donne à mon frère une couleur et après une semaine, il se peut qu'il revienne avec plusieurs thèmes composés, c'est comme un puzzle : on essaie de trouver quel thème va avec quelle scène et avec quelle situation, quel personnage. Voilà le schéma général et un peu simplifié sur la méthode de travail. Concernant l'improvisation, il n'y en a pas beaucoup en tant que telle. Tout 
est très défini : au soir du concert, on sait exactement qu'est-ce qu'on joue à quel moment. Après, durant la scène et pendant ce thème précis, chaque soir est différent parce qu'on ne voit jamais le film de la même manière. Même si on le revoit dix fois en dix jours, il va y avoir dix façons différentes - je parle là des grands films, ceci leur est propre. Et on aime bien le terme de « composition aléatoire ", c'est quelque chose de composé et à l'intérieur, cela va se passer différemment. Peut-être peut-on préciser aussi : comme on est trop proches de l'écran, en fait, on ne le regarde pas en tant que tel, mais on travaille avec des écrans plus petits, des sortes de moniteurs pour permettre malgré tout, pas mal de travail de synchronisation avec l'image. Je pense et cela dépend des scènes, que l'on n'est pas complètement rivés à l'écran. Il y a beaucoup de scènes assez longues, de quinze à vingt minutes, que ce soit dans Faust ou dans Gribiche, où on n'utilise qu'un seul thème, et là c'est typiquement un exemple où la musique n'est pas uniquement présente pour accompagner le film, mais où elle vit par elle-même et se développe en parallèle de l'image. C'est là où la question de l'accompagnement musical est posée. D'un coup on se rend compte - évidemment on a suffisamment vu le film, on a conscience de comment va se développer le thème, avec quelle intensité et quelle vitesse, mais c'est vraiment une évolution parallèle à l'image. Ce sont les moments les plus forts en parallèle, $d$ autant que dans la relation avec mon frère, on échange très peu de regards. On est plongé dans l'obscurité de la salle, dans l'ambiance, on n'a pas vraiment de contact avec le public. On sait qu'il regarde le film, on est vraiment ailleurs parfois, on n'est plus dans la salle, on n'est plus là. Mon frère n'est plus là, on n'est plus ensemble. Il se passe quelque chose d'unique. Je pense que c'est propre au ciné-concert. Parce qu'à un concert "pur », on a beaucoup plus d'échanges avec le public. Là, ce sont plusieurs blocs et plusieurs forces parallèles avec le public, les musiciens et l'image. C'est en cela que ce genre-là est unique. Et aussi en parlant des difficultés, ce n'est pas propre à Faust mais au cinéconcert. Au final on a très peu de recul, en tant que musicien sur ce qui s'est passé : on n'a pas vu le film en entier, pendant qu'on a joué, donc notre perception de la performance est complètement faussée. On sort souvent de là un peu désemparés.

Julia PESLIER - Mettre en musique le Faust de Murnau aujourd'hui signifie en extraire sa part de contemporanéité, d'universalité en même temps que sa singularité et son esthétique. Quelles seraient pour vous l'actualité ou, à rebours, l'inactualité intrinsèques à ce chef d'œuvre? En quoi Faust nous parle-t-il de notre rapport au monde, à l'œuvre d'art, aux langages du diable et aux sphères du savoir?

Bachar KHALIFE - Ce que je peux dire, c'est que ce film, on l'a joué une dizaine de fois, dans des lieux très différents, aussi bien à la Cinémathèque de Paris, au cinéma Balzac qu'à Beyrouth dans la salle Métropolis, à Ankara en Turquie, à l'extérieur dans un festival en Normandie, on l'a joué dans une église, on aurait pu le jouer n'importe où. J'ai l'impression que les gens qui viennent voir Faust savent qu'ils viennent voir quelque chose qui parle du monde, que c'est un thème universel, et en même temps, la part de contemporanéité, elle est totale quand on aborde en Faust la question de faire un choix. La vieillesse... Ou faire le choix de la jeunesse et de passer outre certaines règles morales. Évidemment la religion est abordée d'une manière directe dans ce film. On pourrait peut-être aujourd'hui remplacer la religion par l'argent ou des systèmes de société, les nouveaux modèles de société de consommation. Voilà : dans les détails, on pourrait changer la terminologie des choses, mais on vit encore dans l'époque du Faust de Murnau. Quand la peste débarque et que c'est la panique en ville et qu'on accuse la jeunesse de vouloir s'amuser, c'est le sida des années 1980 , 
c'est la grippe de l'année passée... Et puis j'ai l'impression que c'est traité, assez étonnamment, avec humour. Si on arrive à avoir un peu de recul sur soi, on en rigole parce qu'on se dit qu'il s'est passé cent ans et qu'on a l'impression de ne rien avoir appris. Quant à notre rapport au monde... effectivement, ce film nous invite à avoir un peu de distance avec les événements, avec ce qu'on nous dit. À l'époque, c'était l'église ou ces forces-là, qui distillaient les informations, aujourd'hui les médias ont une force à laquelle on ne peut pas échapper. Une sorte de tyrannie médiatique. Le monde est devenu un petit village: on parle d'un seul sujet comme si tout était rétréci, au lieu d'ouvrir les portes et de voir la beauté où qu'elle se trouve, finalement on referme petit à petit ces portes et on cède à la pensée unique, qui domine et qui triomphe avec ce genre de politique - parce qu'il s'agit bien de politique. Et donc oui, le langage du diable c'est la métaphore, c'est la peur, les instances politiques, leur meilleure arme c'est de faire peur, faire peur aux populations, la loi du marché ça fait peur, on n'y comprend rien mais ça fait peur, on nous dit que le monde s'écroule, que c'est la crise et cela s'oppose aux sphères du savoir. Les sphères du savoir, c'est la science, les arts, mais aussi la poésie, c'est l'amour, le pardon, c'est le droit, la justice, voilà ce sont les sphères du savoir, ce sont les choses qui souffrent le plus et qui souffrent les premières des forces obscures.

Julia PESLIER - Comment comprenez-vous ce propos du réalisateur Éric Rohmer, dans son
analyse passionnante de L'Organisation de l'espace dans le Faust de Murnau: «La puissance
de l'expression plastique prend manifestement le pas sur l'anecdote, en ce drame connu de
tout spectateur. Les contemporains l'ont goûté, et nous le goûtons nous-mêmes comme
une sorte d'opéra visuel, la mise en scène y tenant lieu de partition »? Bachar KHALIFE - Ce livre, je l'ai lu bien après notre travail sur Faust et j'étais content de ne pas l'avoir lu avant. C'est vrai que c'est passionnant, mais je pense que cela aurait faussé, ou en tout cas, cela aurait enlevé beaucoup de la spontanéité. Mais c'est passionnant. J'aime le terme, c'est vrai, de "partition», qu'il utilise dans cette phrase, et non seulement partition, mais opéra, et d'autres termes qui viennent du champ lexical de la musique. Faust, la première fois que je l'ai vu, comme tous les films muets que l'on aborde, c'était en silence. Un film muet peut se regarder en silence et doit pouvoir être vu en silence. Quand j'ai vu Faust sans musique, c'est la première fois que je voyais un film sans musique, car tous les films muets que j'avais vus, c'était du Charlie Chaplin. On a grandi avec ses films, mais Charlie Chaplin était compositeur, c'était sa musique. C'était la première fois que je voyais un film chez moi, seul, sans bruit. La musique ne manque pas. C'est en ce sens que je comprends ce terme d'opéra visuel, et celui de mise en scène qui tient lieu de partition. C'est une œuvre à part entière. Il n'a pas été fait et pensé avec une musique, ce film. Tous les termes de cette phrase, la puissance de l'expression - le film dégage une puissance gigantesque. Et justement le défi du ciné-concert, tel qu'on le conçoit avec mon frère, c'est «comment ne pas nuire à la puissance de l'expression, par exemple, d'un visage? ». Ce n'est pas en plaquant un accord, forcé, que l'expression va être plus significative. L'expression est là, elle est déjà puissante et elle n'a besoin de rien. Donc comment faire avec la musique. C'est pour cela que j'ai parlé d'expression parallèle et pas intrinsèque au film. J'ai pris les termes techniques qui se rapportaient à la musique dans cette citation. On peut la comprendre par rapport à la signification et à la portée de l'œuvre. Mais je pense qu'on en a un peu parlé dans les questions précédentes. 
Julia PESLIER - Dans l'histoire du cinéma muet sonorisé par la performance musicale, la question de l'illustration, «une musique pléonasme» selon Pierre Jansen et Antoine Duhamel (ainsi des accords plaqués pour donner à entendre une porte claquée, des gammes ascendantes pour un escalier gravi) et celle de leitmotiv (une ligne mélodique que l'on attribuait à chaque apparition à l'écran d'un personnage, technique notamment déployée par Ernö Rapee et inspirée de Richard Wagner) ont longtemps fourni un cadre pour le musicien, redoublant l'image par un rapport de redondance. $Y$ avez-vous eu recours d'une manière ou d'une autre ou avez-vous tenu au contraire à proposer d'autres modes de complémentarité, de résonance voire de distanciation entre les images à l'écran, leur rythme et leur montage d'une part et votre double performance musicale de l'autre?

Bachar KHALIFE - C'est toujours d'actualité : on attend toujours cela aujourd'hui et c'est quelque chose de très massif et de difficile à bouger, c'est quelque chose comme une institution, le ciné-concert, c'est la musique qui accompagne le film. C'est pire qu'une institution, ce serait presque une secte, d'une certaine catégorie de spectateurs, cinéphiles, ou amoureux de cinéma. Par exemple, Faust est un chef d'œuvre du cinéma muet: j'ai rencontré certaines personnes qui refusaient catégoriquement l'idée d'un accompagnement musical («c'est un film muet donc il n'y avait pas à mettre de la musique »). Avec d'autres, on a eu des questions après coup : parmi les choses qui retenaient le plus l'attention, il y avait le moment où la fille perd sa mère au début du film, où Faust lance l'antidote par terre et la glace se brise. Comme on avait pris le parti que le verre se brise en silence, cela a retenu beaucoup l'attention, car on attend un son. On est formaté à un certain type de cinéma, et c'était là l'un des défis pour nous. Ce n'est pas le principe de jouer le contraire de ce qui se passe à l'image. Mais on a été confronté à des réactions assez passéistes. Le fait d'utiliser la voix et mêmes certains mots, dans Gribiche, je sais que c'est quelque chose qui n'est pas forcément accepté par les gens qui voient ce concert-là. Mais en même temps, je pense que tout simplement un ciné-concert n'est pas fait pour découvrir forcément un film. Le meilleur public qui puisse venir voir ce type de concert, ce sont soient les enfants, qui n'auront pas encore été formatés à un certain cinéma (hollywoodien notamment), soient des gens qui connaissent parfaitement le film en question, qui en ont saisi la portée et qui viennent le revoir d'une manière différente. Ils savent qu'il y a encore des aspects du film qu'ils n'ont pas encore vus, que certains angles leur ont échappé et que c'est une chance de voir certaines choses jusque-là invisibles à l'écran.

Julia PESLIER - Aujourd'hui, le terme de ciné-concert déplace la sphère du cinéma muet (qu'il soit accompagné musicalement, bruité de diverses manières ou orchestré) vers celle du concert pensé comme l'hybridation entre une œuvre cinématographique classique et une création contemporaine. Dans une perspective traditionnelle, il était souvent question d'un « accompagnement musical », d'une « recréation d'atmosphère » ou « d'ambiance », d'une explicitation sonore afin d'accroître, d'accentuer et de sublimer les effets de l'image en mouvement et les sensations ressenties par le spectateur. Comment définiriez-vous le rapport qui se noue aujourd'hui entre le film muet et la musique dans votre travail ?

Bachar KHALIFE - Je ne sais pas s'il est vraiment approprié : ciné, c'est un peu, presque péjoratif. On va au ciné, on va passer un bon moment - un certain cinéma se prête à cela. Et concert, ce n'est pas du tout un concert. Je ne sais pas si on est obligé de trouver un terme - c'est une forme de spectacle qui est aujourd'hui assez commune, qui se fait un peu partout, mais la définir en ciné-concert... Je pense qu'il y a une mode du ciné-concert et que c'est un peu dangereux pour le genre. Ce que je dis est valable un peu pour tous les autres termes applicables à la musique : quand on dit c'est un concert de musique du monde, c'est vendeur, cela parle tout de suite à une certaine 
catégorie de personnes, mais à partir du moment où on essaie de mettre une étiquette, on perd un peu la notion de cette chose-là. C'est tout simplement un concert. Pour le ciné-concert, il faut peut-être encore un peu de temps pour trouver l'expression la plus juste, la plus adéquate.

Julia PESLIER - Comment votre expérience du ciné-concert vous incite-t-elle à penser le rapport entre le temps cinématographique et le temps musical? Sont-ils différents, conflictuels ou complémentaires? Comment s'articulent-ils, entre le plein et la vitesse des images et le rythme musical, la pause et le silence?

Bachar KHALIFE - Comme je l'ai dit plus haut, on touche à quelque chose d'unique dans ce genre de performance. Quand la scène dure vingt minutes, qu'il y a une image, que les personnages avancent, et qu'il se passe plein de choses et en même temps il ne se passe qu'une seule chose et cette chose unique, c'est comme si le film, l'image - je ne sais pas combien d'images-secondes il faut pour raconter ce qui se passe - comme s'il ne fallait le dire que par la musique et que, par les notes, celles qui constituent le thème, il n'en fallait que deux ou trois. C'est presque jubilatoire. Je pense à la dernière scène qui dure un quart d'heure - il s'y passe beaucoup de choses et en même temps une seule : la fin du film, on la devine, mais on attend toujours l'image pour voir ce qu'il va se passer, la dernière image. C'est un thème unique, deux ou trois notes, pendant un quart d'heure, qui monte en intensité. C'est vrai que le temps n'est pas le même. Et d'ailleurs on finit le thème après l'image. On continue à jouer après l'image, une à deux minutes, après la fin du film, quand l'écran devient noir et que les gens sont prêts à partir, on continue, aussi peut-être pour affirmer qu'il y avait plusieurs forces qui ont travaillé ce soir-là, parfois ensemble, parfois l'une contre l'autre, ou l'une à côté de l'autre et parfois l'une sans l'autre. À la fin, pour nous évidemment, c'est la musique qui triomphe. On prend un peu notre revanche sur le public qui n'avait d'yeux que pour le film. Enfin j'exagère. Cela pose de nombreuses questions, à chaque fois qu'on aborde ce travail. Est-ce qu'on peut se permettre de le faire, est-ce qu'on doit ne pas empiéter sur le film ni sur l'histoire, est-ce qu'on doit être vus, éclairés, dans des lumières ou cachés ? Et plus on en fait, plus on a des questions.

Julia PESLIER - Dans quelle mesure cette performance, qui vous amène à fixer le film de façon continue, afin de synchroniser musique et image, a-t-elle modifié votre regard sur le cinéma?

Bachar KHALIFE - C'est sûr : je pense que je n'ai plus vu un seul film de cinéma de la même manière depuis qu'on a commencé à travailler sur ces films-là. Je ne regarde plus un film pour en saisir l'histoire. Ce n'est plus l'histoire, ni le scénario qui m'intéressent ; ce qui me passionne c'est le cinéma en tant que tel, c'est le plan, c'est le rythme du film. C'est ce que les personnages ne disent pas, ce qu'on ne les voit pas faire, c'est la partie de la maison qui dépasse l'écran. Quand on parle de magie du cinéma, pour moi aujourd'hui, c'est cela. Cela se traduit en tout ce qui est caché. Et c'est de revoir aussi les films dix à quinze fois, avec toujours plus de curiosité, comme si je me rendais totalement à ce qui se passait dans le film, sans résistance.

Julia PESLIER - La pratique du ciné-concert, originale en soi, a-t-elle infléchi en retour votre travail de composition, de création et de concert? Quelles sont les interactions avec vos autres créations artistiques et projets actuels ?

Bachar KHALIFE - C'est l'inverse pour les concerts en tant que tels. Je pense que cela a changé peu de choses, peut-être parce que je suis musicien et que j'ai été formé 
depuis le plus jeune âge à cela. Par contre, dans la composition et dans la manière d'interpréter mes propres compositions, à l'image de la dernière scène de Faust où l'on joue le thème de Faust pendant un quart d'heure, plus je limite les notes, plus je vois un champ d'images infini.

Julia PESLIER - Je souhaiterai revenir sur le rapport à l'historicité et à l'hybridité productive que le ciné-concert créé en intriquant différents temps: celui de l'œuvre cinématographique, déjà classique, son inactualité présente et sa réception qui la remotive par de nouvelles acceptions d'une part et, de l'autre, celui de la performance, en prise avec sa modernité constitutive et sa généalogie musicale, son anachronisme fondamental par rapport au temps de l'œuvre. Y aurait-il, à votre avis, une analogie possible entre le cinéma muet et la performance musicale sans cesse recommencée autour du film et ce que l'on sait de l'œuvre et de sa traduction : à savoir que la première demeure et se déploie sur une longue durée en une infinitude plurielle et que la seconde vieillit, un peu à la manière d'une photographie qui jaunit plus vite que la toile de maître qu'elle représente (pour reprendre la métaphore du traducteur Bernard Lortholary), marquant là sa finitude de manière d'autant plus précieuse qu'elle a conscience de son caractère évanescent, éphémère?

Bachar KHALIFE - Est-ce qu'un ciné-concert peut être un chef d'œuvre à part entière, je ne pense pas. Cela n'enlève en rien à la beauté. Quand j'étais enfant, on nous avait amenés dans une sorte de musée : il y avait un homme assis pas terre et qui dessinait à l'aide d'un bâton sur du sable coloré. Cela faisait deux heures, trois heures. Il avait dessiné quelque chose d'extraordinairement beau, un cavalier, des chevaux, un château, tout cela avec le sable. Et quand tout a été fini, il a pris le bâton à l'horizontal et il a tout effacé, comme cela, avec sa main et c'était pour moi quelque chose de magnifique, de créer quelque chose, d'être dans le beau, et de ne pas l'archiver. Alors qu'aujourd'hui on est effectivement un peu malade d'archive. Un autre exemple me vient, celui d'un grand chef d'orchestre, Celibidache, qui était très réticent à l'idée d'enregistrer des concerts, car pour lui le concert c'est le moment et c'est tout. C'est le moment de voir et d'accepter la beauté. La beauté n'est peut-être qu'éphémère, c'est l'expérience de l'avoir vécue qui reste.

\section{INDEX}

oeuvrecitee Faust (film)

Palavras-chave : acompanhamento musical, performance (arte), filme mudo, adaptação

Palabras claves : acompañamiento musical, cine mudo, adaptación

Keywords : film scores, performance, silent film, adaptation

Mots-clés : cinéconcert, performance, accompagnement musical, cinéma muet, adaptation

\section{AUTEURS}

\section{BACHAR KHALIFÉ}

Compositeur (« Oil Sick », 2010, « Who’s Gonna Get the Ball from Behind... », 2013) et musicien multi-instrumentiste 


\section{JULIA PESLIER}

Université de Franche-Comté. Centre Jacques Petit (ELLIADD)

Maître de conférences en littérature comparée 